\title{
Erratum: Studies of Dust Acoustic Double Layers in the presence of Trapped Particles
}

\author{
[J. Korean Phys. Soc. 64, 232 (2014)] \\ M. K. Mahanta, ${ }^{*}$ R. Moulick and K. S. Goswami \\ Center of Plasma Physics-Institute for Plasma Research, Guwahati, India
}

DOI: $10.3938 /$ jkps.64.619

Affiliation of M. K. MAhanta is written as "Centre of Plasma Physics-Institute for Plasma Research, Guwahati, India". But it should be written as "Arya Vidyapeeth College, Guwahati, India, and Centre of Plasma Physics-Institute for Plasma Research, Guwahati, India". 